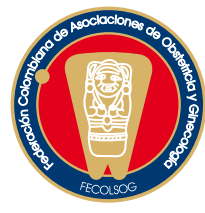

Artículo DE REFLEXIón

\title{
¿ES EL MOMENTO DE VACUNAR CONTRA EL VIRUS DEL PAPILOMA HUMANO EN COLOMBIA?
}

\section{Is it time to vaccinate against human papilloma vinus in Colombia?}

Mario Arturo González-Mariño, M.D.*

Recibido: octubre 14/09 - Aceptado: abril 26/10

\section{RESUIMEN}

Objetivo: analizar el estado actual de la evidencia de la vacunación para el virus de papiloma humano (VPH) a nivel poblacional teniendo como referente su posible aplicación en Colombia.

Metodología: evaluación de estudios fase III sobre las vacunas contra el VPH: estudios de los grupos FUTURE I, II y PATRICIA. Se reflexiona sobre su generalización a nivel poblacional y se analizan factores que limitan su uso.

Resultados: la evaluación de estos estudios encuentra limitaciones metodológicas que controvierten la eficacia presentada en las publicaciones de los estudios como son la elección de variables sustitutas inadecuadas, análisis de resultados con variables primarias compuestas que son heterogéneas, análisis por protocolo y no por intención de tratamiento, criterios de inclusión que no sustentan la aplicación poblacional de la vacuna en menores de 15 años y factores que pueden afectar la validez de los estudios como conflictos de intereses y origen del patrocinio. De otra parte, se presentan reportes de complicaciones con la aplicación de la vacuna que brindan una mejor información sobre el verdadero comportamiento de este método de prevención por fuera del diseño experimental.

* Médico Ginecólogo y Oncólogo. Profesor Catedrático Asociado, Facultad de Medicina, Universidad Nacional de Colombia. Profesor, Facultad de Medicina, Fundación Universitaria San Martín. Médico, Hospital Central de la Policía y Hospital Militar Central. Bogotá (Colombia).Correo electrónico: marioar90@hotmail.com
Conclusiones: para el momento de esta revisión no se tiene la suficiente evidencia que sustente la inclusión de la vacuna contra el VPH como programa de vacunación poblacional en Colombia.

Palabras clave: cuello del útero, cáncer de cuello uterino, cérvix uterino, infecciones por virus, virus del papiloma humano 16, virus del papiloma humano 18 , prevención primaria.

\section{SUIMMARY}

Objective: analysing current evidence regarding proposals for vaccinating the Colombian population against human papilloma virus (HPV).

Methodology: assessing HPV vaccine phase III studies (FUTURE I, II and PATRICIA study groups). Comments are made regarding generalising such vaccination amongst the general population, also taking factors limiting their use into consideration.

Results: methodological limitations were found when assessing these studies thereby challenging the importance of the efficacy found in such studies such as the choice of inadequate substitutes, analysing results involving heterogeneous primary compounds, analysis by protocol and not by intention to treat, inclusion criteria which did not underpin a general vaccination programme for girls aged younger than 15 and factors which could affect the validity of the studies such as conflicts of interests and sources of sponsorship. Several complications have been reported regarding how the vaccine has 
been applied, providing better information about this prevention method's real behaviour (i.e. when taken beyond its experimental design stage).

Conclusions: there was not sufficient evidence to recommend introducing HPV vaccination within Colombian immunisation schedules when this review was made.

Key words: uterine cervix, cervical cancer, human papilloma virus 16 , human papilloma virus 18 , primary prevention.

\section{INTRODUICCIÓN}

El cáncer de cuello uterino es el cáncer más frecuente en Colombia con una incidencia ajustada de 36,8 por 100.000 por lo que se ubica entre los países de alto riesgo para sufrir esta enfermedad. El desarrollo de esta neoplasia está estrechamente relacionado con el virus del papiloma humano (VPH) cuya infección persistente con uno de los virus de alto riesgo se considera causa necesaria en el cáncer de cérvix. ${ }^{2,3}$ La infección por el virus del papiloma humano es la más frecuente entre las enfermedades de transmisión sexual en el mundo. ${ }^{4}$ En EE.UU. se calcula que el $80 \%$ de los hombres y mujeres se infectarán con el VPH en algún momento de su vida. ${ }^{5}$ Dicha infección transcurre la mayoría de las veces sin contratiempos gracias a que el sistema inmune logra su control sin dejar secuelas clínicas ${ }^{6,7}$ y solamente en un porcentaje pequeño (10\%) se da la situación en que la infección persiste. ${ }^{8}$ Se consideran virus de alto riesgo los siguientes: 16, 18, 31, 33, 35, 39, 45, $51,52,56,58,59,66,68$, y $73^{3}$ siendo el tipo 16 al que se le encuentra con mayor frecuencia en los carcinomas [50\% en los cánceres y en el NIC (neoplasia intraepitelial cervical) de alto grado $^{9-11}$ y en el 25\% de los de bajo grado]. ${ }^{9,10}$ En combinación con el virus VPH 18, intervienen en la carcinogénesis en cerca del 70\% de los cánceres invasivos de cérvix. ${ }^{12}$ En el desarrollo a cáncer de cérvix, además se da la presencia de otros factores no bien conocidos. ${ }^{7}$ Se calcula que en aquellas que hacen cambios celulares correspondientes a
NIC 1, el 1\% pueden desarrollar cáncer invasivo de cérvix, las de NIC 2 lo van a hacer en el 5\% y las de NIC 3 en más del 12\% cuando no reciben tratamiento. ${ }^{6}$ Esto significa que el cáncer de cérvix se desarrolla en una minoría de mujeres aún en ausencia de pruebas de cribado.

La asociación entre el cáncer de cérvix y el virus del papiloma humano hace que el desarrollo de las vacunas sea un avance notable en la lucha contra esta enfermedad. Sin embargo al momento existen vacíos en el conocimiento al respecto, como son la duración de la protección, la necesidad de refuerzos, marcadores inmunológicos de protección, eficacia en los hombres y en las mujeres mayores, seguridad a largo plazo, inmunogenicidad en la niñez, eficacia con menos de tres dosis, desarrollo de nuevas vacunas con menor costo y con mayor número de tipos virales, efecto en los programas de tamización ${ }^{13,14}$ y evidentemente, su impacto en el desarrollo del cáncer invasivo de cérvix y en su consecuente mortalidad.

Este documento tiene como objetivo analizar la evidencia de la vacunación para el virus de papiloma humano (VPH) a nivel poblacional teniendo como referente su posible aplicación en Colombia.

\section{METODOLOGÍA}

Evaluación de los estudios de las vacunas contra el VPH fase III en los grupos FUTURE I, II y PATRICIA. Se reflexiona sobre su generalización a nivel poblacional y se analizan factores que limitan su uso.

\section{DISCUISIÓN}

\section{FUTURE I}

En el estudio FUTURE I ${ }^{15}$ se incluyeron mujeres de 16 a 24 años. La tasa de incidencia de NIC 1 a 3 o de adenocarcinoma in situ por 100 personas-año fue de 4,7 en mujeres vacunadas y 5,9 en mujeres no vacunadas, con una eficacia del 20\%. El análisis según el tipo de lesión muestra que esta disminución se debe fundamentalmente a una menor frecuencia de NIC 1 en las pacientes vacunadas; no se encontró una mayor eficacia para el de alto grado pero puede 
ser porque la prueba carecía del poder adecuado para encontrar la diferencia. ${ }^{16}$

\section{FUTURE II}

En el FUTURE II $^{17}$ se presentan datos de la vacuna tetravalente con relación a la protección de patología cervical en pacientes de 15 a 26 años, sin evidencia virológica de la enfermedad hasta el séptimo mes (un mes después de la tercera dosis) mediante un análisis primario por intención de protocolo y con múltiples resultados primarios que incluyen NIC 2 y 3 , adenocarcinoma in situ y carcinoma invasivo, en los cuales se detectó ADN de VPH 16 ó 18. La proporción de grado 2, 3 o adenocarcinoma in situ fue de 1,3 en las mujeres vacunadas y de 1,5 en las no vacunadas con una eficacia del 17\%. En el análisis por el tipo de lesión la eficacia es significativa solamente para el NIC 2, no para el NIC 3 ni para el adenocarcinoma in situ. ${ }^{16} \mathrm{Al}$ tomar estas tres patologías, la diferencia en el riesgo parece modesta con 3,6\% en las vacunadas y 4,4\% en las no vacunadas, (la diferencia absoluta del riesgo es de $0,8 \%$ ) lo que implica que se requiere vacunar 129 mujeres para prevenir un caso de NIC de alto grado o de adenocarcinoma in situ en un período de 3 años. El resultado global compuesto (NIC 2 ó 3, adenocarcinoma in situ o carcinoma invasivo con ADN de VPH 16, 18 o ambos, en uno o más de tres cortes adyacentes de la misma lesión) en eficacia fue de 98\% (IC95,89\% 86-100) en el análisis por protocolo pero en el análisis por intención de tratamiento fue de 44\% (IC95\% 26-58). ${ }^{17}$

Jorma Paavonen y colaboradores, presentaron en el año 2007 un informe preliminar de un estudio fase III que evaluó la eficacia de la vacuna de VPH 16/18 en mujeres de 15 a 25 años. ${ }^{18,19}$ En la publicación final de este estudio en el mes de julio de 2009, se presentan los análisis por intención de protocolo en los que la eficacia de la vacuna fue 92,9\% (IC96,1\% 79,9-98,3), en el análisis según intención de tratar fue 30,4\% (16,4-42,1) y 70,2\% (54,7-80,9) en las participantes que al ingreso tenían citología normal, eran negativas en el análisis de ADN para los 14 tipos de VPH oncogénicos evaluados y eran sero-negativas para los tipos 16 y $18 .{ }^{20}$ Hay varios factores que comprometen la generalización de los resultados de este estudio comenzando por lo restringido de los criterios de inclusión y posteriormente para el análisis de inmunogenicidad (12\% de los participantes). También el bajo número de mujeres hispanas y negras. ${ }^{18}$

\section{EDAD DE VACUNACIÓN}

Como se puede apreciar en los estudios mencionados, ninguno de estos incluye niñas menores de 15 años y si bien hay pruebas pequeñas en estas condiciones, estas carecen de la trascendencia y el valor investigativo para servir de base a entidades internacionales y a varios países para hacer recomendaciones de vacunación en menores de 15 años. ${ }^{21,22}$ Surge aquí una extraña posición en estos organismos al recomendar la vacuna en edades que los estudios relevantes no han contemplado como criterio de inclusión y que por lo tanto no ha sido evaluada ni en su eficacia ni tampoco en sus riesgos. Llama la atención que en publicaciones recientes también financiadas por la industria farmacéutica, afirmen que se ha probado su beneficio en las edades de 9 a 26 años $^{23}$ citando los estudios arriba mencionados, FUTURE I y II, cuando está claro que la recomendación de vacunar niñas y adolescentes tempranas (12 a 14 años) no puede surgir de estos estudios. ${ }^{16}$

La febril actividad de introducir la vacuna en niñas se contrapone a la recomendación epidemiológica de extrapolar los resultados esencialmente a individuos con las características de la muestra evaluada, ${ }^{24}$ por consiguiente carecemos de buena información sobre el desempeño de esta vacuna en el grupo etario en el cual se está recomendando de manera prolífica su aplicación.

\section{TAMAÑO DE LA MUESTRA}

La efectividad con la cual las vacunas profilácticas consiguen reducir el NIC 3 en la población general se tiene que inferir con base en estos estudios con 
muestras pequeñas. En las pruebas de la vacuna tetravalente, precisamente debido al tamaño de la muestra, la incidencia de NIC 3 (independiente del tipo de VPH) fue cercana entre los grupos comparados (0,7 en las vacunadas y 0,9 en las de placebo por 100 mujeres). ${ }^{25}$

\section{SEGUIMIENTO}

$\mathrm{Al}$ revisar los estudios sobre las vacunas, una apreciación es que la duración del seguimiento en estos es corta teniendo en cuenta que la carcinogénesis cervical usualmente se desarrolla a través de varias décadas. ${ }^{26}$ Actualmente, no es posible predecir exactamente el efecto que la vacunación tendrá en la incidencia del cáncer cervical a desarrollar 20 a 40 años después, ${ }^{27}$ pero se estima que los países desarrollados tendrán que esperar 30 años para observar una reducción del 20\% en la incidencia del cáncer de cérvix contando con una cobertura por la vacuna del $75 \%$ en la población..$^{28} \mathrm{Al}$ respecto, Harper comenta que los estudios de costo-efectividad predicen una reducción en el cáncer de cérvix dentro de varias décadas, pero solamente cuando el 90\% de todas las niñas de 11 a 12 años hayan sido vacunadas por varios años asumiendo una protección de por vida; ${ }^{29}$ si la protección es menor a 15 años, la incidencia del cáncer de cérvix se presentará en mayores de 25 años, sin que ocurra disminución del cáncer con el uso de la vacuna, haciendo a estas mujeres susceptibles a los tipos de VPH oncogénicos. ${ }^{30}$

El verdadero efecto de la vacuna se determinará solamente mediante pruebas clínicas y esencialmente a través de un seguimiento juicioso a través de los años. ${ }^{27}$ Este seguimiento permitirá además valorar la duración de la protección que las vacunas puedan conferir dado que es improbable que con su aplicación, por ejemplo a los 12 años, confiera inmunidad de por vida siendo lo esperado que se requiera de un refuerzo. Teniendo en cuenta que las infecciones persistentes son más frecuentes en mujeres con edades mayores a las valoradas en estos estudios, en individuos con función inmune alterada y que además, se ha encontrado un segundo pico en la prevalencia del VPH en las mujeres en la cuarta y quinta décadas de la vida ${ }^{31}$ serían estas mujeres las de mayor riesgo de cáncer invasivo de cérvix y por tanto las que la vacuna debería proteger, de lo contrario la incidencia de cáncer de cérvix se podría incluso incrementar. Esto además, recalca la necesidad de fortalecer los programas de tamizaje, que permitan un seguimiento confiable de estas mujeres. ${ }^{32}$

Un seguimiento prolongado de las pacientes vacunadas puede dar lugar a cambios con respecto a la valoración en el estudio de la vacuna como modificaciones en el nivel socio-económico, mejoras en los programas de cribado, hallazgo de marcadores moleculares que permitan una mejor prevención secundaria de la enfermedad, todos ellos factores que pueden confundir los resultados del impacto de la vacunación. También pueden cambiar los mismos factores de riesgo, como el cambio en la prevalencia de los tipos virales de VPH ya sea de manera espontánea ${ }^{33}$ o relacionada con la vacunación, generándose potencialmente otros virus de alto riesgo. ${ }^{25}$

\section{VARIABLES SUSTITUTAS}

Frecuentemente en la evaluación final de una investigación no se usan puntos primarios como la sobrevida sino sustitutos, ello por razones como el ahorro de costos a las empresas farmacéuticas, mejoría en la viabilidad de realización de pruebas clínicas porque agilizan el proceso de aprobación de medicamentos y su disponibilidad en el mercado. ${ }^{34}$

Sin embargo, hay que tener en cuenta que por la misma razón de reemplazar los puntos primarios, los estudios que se miden con variables sustitutas generalmente son de menor evidencia. ${ }^{35}$

Las variables sustitutas deben cumplir con ciertos requerimientos para que sean útiles y representativas del resultado primario esperable pero, básicamente, deben predecir el efecto del tratamiento en el riesgo del resultado clínico primario. $^{36}$ 
Para la valoración de la vacunación, se han usado variables sustitutas tales como los niveles serológicos de anticuerpos para los tipos de virus del papiloma humano presentes en la vacuna, con lo cual se busca medir la respuesta inmunológica. ${ }^{37}$ En el caso de los estudios fase III se evalúan los siguientes resultados: FUTURE I, se valoraron como resultados "primarios compuestos" en la patología cervical: el NIC 1 a 3, adenocarcinoma in situ y cánceres asociados con los tipos 6, 11, 16 ó 18. El seguimiento se hizo durante un tiempo promedio de tres años medido desde la primera dosis. ${ }^{15}$ FUTURE II analizó un resultado primario compuesto de NIC 2 y 3, adenocarcinoma y cáncer relacionados con los virus VPH 16 y 18, con un seguimiento promedio de tres años desde la aplicación de la primera dosis de la vacuna o de placebo. ${ }^{17}$ El estudio PATRICIA evaluó la eficacia de la vacuna contra NIC 2 y 3 asociadas con VPH 16 y 18; también se evaluaron de manera secundaria las infecciones persistentes a los 6 y 12 meses. El tiempo medio en la cohorte seguida durante más tiempo fue 39,4 meses. ${ }^{22}$

El uso de estos resultados permite analizar: si las vacunas se plantean a nivel de mercadeo para la prevención del cáncer de cérvix, entonces la inclusión del NIC 1 como un resultado primario, que se evalúa al mismo nivel que el cáncer invasivo en FUTURE I, es inadecuado ya que a esta lesión no se le considera precancerosa. ${ }^{16}$ En los NIC 2 y 3, si bien se contempla un tratamiento semejante en las mujeres adultas, el comportamiento entre estas dos lesiones ofrece diferencias; así, se calcula que el 40\% de los NIC 2 pueden remitir espontáneamente ${ }^{38,39}$ dando lugar a que su inclusión realmente no valore el potencial de prevención del carcinoma invasivo ya que no guarda una conexión directa inmediata con éste, por lo que difícilmente se le puede aceptar como una variable sustituta. Hay que tener en cuenta que los estudios mencionados arriba son pruebas clínicas controladas con un laboratorio central que pretende evitar la variabilidad interobservador y con controles periódicos dentro del marco del estudio que permite que una vez se haya diagnosticado NIC
2, se valore su persistencia y posible progresión mas no un uso poblacional en el cual este seguimiento puede resultar más difícil.

La observación es el enfoque de elección en el manejo clínico del NIC 2 cuando se encuentra en menores de 20 años. ${ }^{40}$ Esto para señalar que en los estudios no debió evaluarse el NIC 2 como variable primaria y tampoco indicar un tratamiento probablemente innecesario a las participantes, sino que se debieron evaluar solamente aquellas pacientes que en el seguimiento progresaron a NIC 3.

El NIC 3, si bien se considera como el mejor resultado sustituto, al ser considerado como un precáncer puede estar compuesto por alteraciones de comportamiento heterogéneo, lo que daría lugar a que algunos puedan tener un mejor pronóstico. De esta forma, se supone que no es igual un NIC 3 encontrado en una prueba clínica al que se encuentra después de varios años en un examen de consulta rutinaria. ${ }^{41}$

La relación entre el VPH y el adenocarcinoma in situ, si bien parece conservar la causalidad necesaria del exocervical, tiene una peor evidencia en estudios epidemiológicos con menor tamaño de la muestra, poca información sobre la distribución geográfica y en los que se conocen menos los cofactores. ${ }^{42}$

En los estudios FUTURE los desenlaces denominados primarios son en la práctica sustitutos (no se reporta cáncer invasivo) y son analizados de manera compuesta. Estos resultados compuestos se aceptan bajo la premisa que el efecto de la vacuna será semejante entre los componentes (por ejemplo en los NIC 1, 2 y 3 o en el adenocarcinoma in situ) y que las pacientes valoran como de igual importancia a cada uno de los componentes, ${ }^{43}$ lo cual no se supone que ocurra en este caso dado que incluye elementos con gran diferencia en su comportamiento, que por supuesto no tienen el mismo valor dentro de lo que se espera que la vacuna brinde como factor protector, esto es, para el cáncer invasivo y además, como se presentó previamente, los resultados descansan de manera importante en las respuestas favorables en las lesiones de NIC 1 y $2{ }^{44}$ 


\section{TIIPOS DE ANÁLISIS}

En los estudios experimentales se recomienda que el análisis sea por intención de tratar, es decir, en el que los sujetos son analizados dentro de los grupos a los que inicialmente fueron asignados, independientemente de si recibieron la intervención, conservando así los beneficios de la asignación aleatoria. ${ }^{45}$

El análisis por protocolo (FUTURE I y II) incluye sólo a los participantes en los que se pudo medir la variable de respuesta y que cumplieron adecuadamente la intervención que les fue asignada. Este tipo de análisis puede presentar sesgo de manera importante, lo que puede sobreestimar el efecto del tratamiento. ${ }^{46,47}$ Existen ensayos que muestran resultados beneficiosos de una intervención al analizar sólo los pacientes que completan o se adhieren al protocolo (análisis por protocolo), sin embargo, al analizar por intención de tratar, los beneficios no son tan evidentes. ${ }^{48}$

En el análisis por intención de tratar se da una estimación en la vida real del beneficio de la vacuna más que del beneficio potencial en las participantes que recibieron el tratamiento exactamente como se planeó dado que, por ejemplo, es común que en la práctica médica ocurra la falta de adherencia al tratamiento. ${ }^{49}$

\section{FINANCIACIÓN \\ DE LOS ESTUDIOS}

Dentro de la intención de evaluar poblacionalmente la vacunación, se debe tener en cuenta que los estudios existentes han sido diseñados, financiados, evaluados o publicados con patrocinio de los laboratorios productores de las vacunas. En los estudios, participaron investigadores de primera línea, algunos con acciones en la respectiva compañía (no está claro si las pacientes en el estudio sabían de estas relaciones financieras de los investigadores lo que podría haber motivado su libre decisión de no continuar en el estudio), ${ }^{50}$ se contó con el acompañamiento y la participación activa de entidades gubernamentales (de las cuales se desearía que tuvieran la posibilidad de una investigación independiente) y de sociedades científicas, algunas de las cuales no tenían vínculo o interés previo en los programas de vacunación pero que se integraron a programas de mercadeo de la compañía farmacéutica promoviendo la vacuna desde antes que se conocieran los resultados de la investigación. $^{27}$

Respecto a los estudios patrocinados por laboratorios farmacéuticos se ha encontrado que tienden a ser más favorables al patrocinante que los resultados obtenidos por otros tipos de patrocinio y que los efectos adversos son menos frecuentes en los estudios con financiamiento de la industria que en aquellos que no lo tienen. ${ }^{51}$ En una revisión que analiza estudios de metaanálisis de medicamentos por parte de la industria farmacéutica, con aquellas realizadas por Cochrane, se llama a tener cuidado con los primeros por tener menos transparencia, menos reservas con las limitaciones metodológicas y conclusiones mas favorables que las de Cochrane. ${ }^{52}$

Dibarbora presenta el llamado "sesgo de patrocinio" de la siguiente manera: «usualmente los profesionales locales no intervienen en las primeras etapas de la investigación, sino que el protocolo les llega "armado", generado casi totalmente en la empresa farmacéutica que lo considera su propiedad, que no admite ser modificado sustancialmente y tampoco accede al pedido de demasiadas aclaraciones. Así, vienen impuestos: el diseño, los objetivos, la metodología, los aspectos éticos, los criterios de inclusión y exclusión, el consentimiento informado y las indicaciones para ejecutarlo (con traducciones muchas veces deficientes que lo tornan incomprensible para la mayoría de las personas, aún para las versadas en el tema), las pautas de reclutamiento de los sujetosprobandos de una experimentación, etc.». ${ }^{53}$

"Frente a los patrocinadores multinacionales la contraparte local son los investigadores cuyas tareas, básicamente, se limitan a ensayar con pacientes que tienen la enfermedad de interés para la empresa farmacéutica y que se aprovechan en muchos casos en forma inequitativa de la infraestructura de los efectores de la salud pública en la que se desempeñan y no siempre compensando los gastos extras 
que agregan a los ya escasos recursos destinados para atender la salud de la población”. ${ }^{33}$

\section{EFECTOS ADVERSOS}

\section{DE LA VACUNA}

En un artículo publicado en la revista JAMA, Slade y sus colegas, pertenecientes al Centro para el Control de Enfermedades de los Estados Unidos y a la FDA, publican los efectos adversos de la vacuna tetravalente según el sistema de reporte de efectos adversos de las vacunas en los Estados Unidos (VAERS, por sus siglas en inglés) entre junio 1 de 2006 y diciembre 31 de 2008. Aunque la mayoría de los casos son leves, hubo sin embargo algunas reacciones de hipersensibilidad como anafilaxia, síndrome de Guillain-Barré, mielitis transversa, enfermedad de la neurona motora, tromboembolismo venoso y se reportaron 32 muertes. Puesto que el VAERS es un sistema de reporte pasivo y voluntario, los autores sugieren la realización de estudios adicionales, pero por ahora es difícil concluir que la vacuna no tenga efectos serios. Por lo pronto, este reporte sirve para a ayudar a los padres de las niñas en la decisión sobre si aplicar una vacuna para evitar la ocurrencia incierta de un carcinoma o exponer a sus hijas a los más cercanos riesgos de complicaciones por la vacuna. ${ }^{54}$

\section{CONSIIDERACIONES FINALES}

La aplicación de vacunas en la prevención del cáncer de cérvix, aunque llamativamente simple, resulta muy atractiva: si se puede prevenir el VPH, no va aparecer el cáncer, pero en la práctica la situación es más compleja. Hay más de 100 tipos diferentes de VPH y por lo menos 15 son oncogénicos; la relación entre infección en la mujer joven y el desarrollo de cáncer 20 a 40 años después no se conoce; surgen dificultades para la inclusión en programas nacionales de vacunación e inquietudes de los padres con respecto a este tipo de vacunación; se ignora la duración de la protección y la necesidad de dosis de refuerzo en cuyo caso se anticipa que no se va a lograr la misma participación de las mujeres, ${ }^{55,56}$ dando lugar a que estando vacunadas sean susceptibles a los tipos virales de la vacuna; se requiere mayor conocimiento sobre la seguridad de la vacuna; pueden aparecer cambios en la distribución de los tipos virales oncogénicos; ${ }^{57}$ etc. Asimismo, pueden presentarse dificultades logísticas en cuanto a que la mayoría de los países en desarrollo no vacunan a los niños mayores ni a los adolescentes, creando la necesidad de incluir esta población para la vacuna del $\mathrm{VPH}^{32}$ con el propósito de realizar una vacunación general, evitando los esfuerzos aislados.

El costo de la vacuna y el de montar la infraestructura son obstáculos significativos para su uso amplio. Además están otros costos entre los que se encuentran los del sistema de salud, salarios, entrenamiento, transporte, manejo de la cadena de frío, jeringas y su disposición segura, educación e información a la comunidad. A futuro, se debe garantizar la sostenibilidad de la vacunación sopesando esta inversión con respecto al uso de estos recursos en otras vacunas, ampliando la cobertura de las ya implementadas o en programas de detección y tratamiento. ${ }^{57}$

La vacunación representa un mecanismo de prevención de enfermedad muy adecuado, sin embargo, no hay suficiente evidencia que permita recomendar a nivel poblacional el uso de las vacunas para el VPH, que por lo demás, tampoco llevan el tiempo suficiente para demostrar su efectividad en esta enfermedad por lo que parece poco probable que se pueda esperar éxito en la salud pública inmunizando a las niñas y mujeres adolescentes para prevenir el cáncer de cérvix que las puede afectar después de varias décadas. ${ }^{58}$ El modelo de causalidad del VPH como causa necesaria del cáncer de cérvix aunque atractivo, impone que se evalúen con más detenimiento los cofactores que hacen que siendo una infección con alta prevalencia, sea relativamente bajo el número de mujeres que desarrollan el cáncer.

\section{CONCLUSIONES}

La vacunación del virus del papiloma humano es un avance importante en salud pública que busca 
la prevención del cáncer de cuello uterino. Sin embargo, para el momento de esta revisión, aunque algunos países han implementado programas poblacionales, no se tiene la suficiente evidencia que recomiende su inclusión a nivel de la población colombiana. Se requieren esfuerzos que continúen la valoración de estas alternativas de prevención y dentro del futuro de las vacunas con finalidad terapéutica, crear y mejorar programas de registro, tanto del cáncer como de la vacunación, y continuar el fortalecimiento de la prevención secundaria con la posibilidad de evaluación y tratamiento de las citologías anormales.

\section{REFERENCIAS}

1. Ferlay J, Bray F, Pisani P, Parkin DM. GLOBOCAN 2002: Cancer incidence, mortality and Prevalence worldwide, Version 2.0. IARC Cancer Base No. 5. Lyon: IARC Press;2004.

2. Walboomers JMM, Jacobs MV, Manos MM, Bosch FX, Kummer JA, Shah KV, et al. Human papillomavirus Is a necessary cause of invasive cervical cancer worldwide. J Pathol 1999;189:12-9.

3. Parkin DM, Almonte M, Brunic L, Cliffordd G, Curado MP, Piñeros M. Burden and trends of typespecific human papillomavirus infections and related iseases in the latin america and Caribbean Region. Vaccine 2008;26:L1-15.

4. Committee on Adolescent Health Care. ACOG Committee Opinion No. 436: evaluation and Management of abnormal Cervical Cytology and Histology in Adolescents. Obstetrics and Gynecology 2009;113:1422-5.

5. Koutsky L. Epidemiology of genital human papillomavirus infection. Am J Med 1997;102:3-8.

6. Pinto AP, Crum CP. Natural history of cervical neoplasia: defining progression and its consequence. Clin Obstet Gynecol 2000;43:352-62.

7. Schiffman M, Castle PE, Jeronimo J, Rodriguez AC, Wacholder S. Human papillomavirus and cervical cancer. Lancet 2007;370:890-907.

8. Elfgren K, Kalantari M, Moberger B, Hagmar B, Dillner J. A population-based five-year follow-up study of cervical human papillomavirus infection. Am J Obstet Gynecol 2000;183:561-7.
9. Bosch FX, Manos MM, Munoz N, Sherman M, Jansen AM, Peto J, et al. Prevalence of human papillomavirus in cervical cancer: a worldwide perspective. J Natl Cancer Inst 1995;87:796-802.

10. Kulasingam SL, Hughes JP, Kiviat NB, Mao C, Weiss NS, Kuypers JM, et al. Evaluation of human papillomavirus testing in primary screening for cervical abnormalities: comparison of sensitivity, specificity, and of referral. JAMA 2002;288:1749-57.

11. The Atypical Squamous Cells of Undetermined Significance/Low-Grade Squamous Intraepithelial Lesions Triage Study (ALTS) Group. Human papillomavirus testing for triage of women with cytologic evidence of low-grade squamous intraepithelial lesions: baseline data from a randomized trial. J Natl Cancer Inst 2000;92:397-402.

12. Koutsky L. The epidemiology behind the HPV vaccine discovery. Ann Epidemiol 2009;19:239-44.

13. Ministerio de la Protección Social, Instituto Nacional de Cancerología. Recomendaciones para la tamización de neoplasias del cuello uterino en mujeres sin antecedentes de patología cervical (preinvasora o invasora) en Colombia. Guía de práctica clínica (No. 3). Bogotá, diciembre de 2007.

14. Muñoz N, Franco EL, Herrero R, Andrus JK, de Quadros C, Goldie SJ, et al. Recommendations for cervical cancer prevention in Latin America and the Caribbean. Vaccine 2008 ;26S:96-107.

15. Garland SM, Hernandez-Avila M, Wheeler CM, Perez G, Harper DM, Leodolter S, et al. Quadrivalent vaccine against human papillomavirus to prevent anogenital diseases. N Engl J Med 2007;356:1928-43.

16. Sawaya G, Smith-McCune K. HPV Vaccinationmore answers, more questions. N Engl J Med 2007;356:1991-3.

17. FUTURE II Study Group. Quadrivalent vaccine against human papillomavirus to prevent high-grade cervical lesions. N Engl J Med 2007; 356:1915-27.

18. Kahn JA, Burk RD. Papillomavirus vaccines in perspective. Lancet 2007; 369:2135-7.

19. Paavonen J, Jenkins D, Bosch FX, Naud P, Salmerón J, Wheeler CM, et al. Efficacy of a prophylactic adjuvanted bivalent L1 virus like particle vaccine against infection with HPV 16 and 18 in young women: an interim analysis of a phase III double blind, randomized controlled trial. Lancet 2007;369:2161-70. 
20. Paavonen J, Naud P, Salmerón J, Wheeler CM, Chow SN, Apter D, et al. Efficacy of human papillomavirus (HPV)-16/18 AS04-adjuvanted vaccine against cervical infection and precancer caused by oncogenic HPV types (PATRICIA): final analysis of a double-blind, randomised study in young women. Lancet 2009;374:301-14.

21. Pederson C, Petaja T, Strauss G, Rumke HC, Poder A. inmunization of early adolescent females with human papillomavirus type 16 and $18 \mathrm{~L} 1$ virus-like particle vaccine containing ASO4 adjuvant. J Adolesc Health 2007;40:564-71.

22. Reisinger KS, Block SL, Lazcano-Ponce E, Samakoses R, Esser MT, Erick J, Puchalski D, Giacoletti KE, Sings HL, Lukac S, Alvarez FB, Barr E. Safety and persistent immunogenicity of a quadrivalent human papillomavirus types $6,11,16,18 \mathrm{~L} 1$ virus-like vaccine in preadolescents and adolescents: a randomized controlled trial. Pediatr Infect Dis J 2007;26:201-9.

23. Muñoz N, Manalastas Jr R, Pitisuttithum P, Tresukosol D, Monsonego J, Ault K, et al. Safety, immunogenicity, and efficacy of quadrivalent human papillomavirus (types $6,11,16,18$ ) recombinant vaccine in women aged 24-45 years: a randomised, double-blind trial. Lancet 2009;373:1949-57.

24. Riegelman RK, Hirsch RP. Cómo estudiar un estudio y probar una prueba: lectura crítica de la literatura médica. Segunda Edición. (Publicación Científica No 531). Washington DC: Organización Panamericana de la Salud; 1992. p. 260.

25. Chang Y, Brewer NT, Rinas AC, Schmitt K, Jennifer S. Smith JS. Evaluating the impact of human papillomavirus vaccines. Vaccine 2009;27:4355-62.

26. Michel KB, zur Hausen H. HPV vaccine for all. Lancet 2009;374:268-70.

27. Haug C. The risks and benefits of HPV vaccination. JAMA 2009;302:795-6.

28. Plummer M, Franceschi S. Strategies for HPV prevention. Virus Res 2002;89:285-93.

29. Harper DM. Preliminary HPV vaccine results for women older than 25 years. Lancet 2009;373:1921-2.

30. Trottier H, Mahmud S, Prado JC, Sobrinho JS, Costa MC, Rohan TE, et al. Type-specific duration of human papillomavirus infection: implications for human papillomavirus screening and vaccination. J Infect Dis 2008; 197:1436-47.

31. De Sanjose S, Diaz M, Castellsague X, et al. Worldwide prevalence and genotype distribution of cervical human papillomavirus DNA in women with normal cytology: a meta-analysis. Lancet Infect Dis 2007:453-9.

32. World Health Organization. Cervical cancer, human papillomavirus (HPV), and HPV vaccines-Key points for policy-makers and health professionals. WHO, PATH, UNFPA; 2007.

33. Wheeler CM, Hunt WC, Joste NE, Key CR, Quint WG, Castle PE. Human papillomavirus genotype Distributions: Implications for vaccination and cancer screening in the United States. J Natl Cancer Inst 2009; 101:475-87.

34. Shi Q, Sargent DJ. Meta-analysis for the evaluation of surrogate endpoints in cancer clinical trials. Int J Clin Oncol 2009;14:102-11.

35. GRADE Working Group. Grading quality of evidence and strength of Recommendations. BMJ 2004;328:1490.

36. Bell KJL, Irwig L, Craig JC, Macaskill P. Use of randomised trials to decide when to monitor response to new treatment. BMJ 2008;336:361-5.

37. Pedersen C, Petaja T, Strauss G, Rumke HC, Poder A, Richardus JH, et al. Inmunization of early adolescent Females with human papilomavirus type 16 and $18 \mathrm{~L} 1$ virus-like particle vaccine containing ASO4 adjuvant. J Adolescent Health 2007;40:564-71.

38. ASCUS-LSIL Triage Study (ALTS) Group. Results of a randomized trial on the management of cytology interpretations of atypical squamous cells of undetermined significance. Am J Obstet Gynecol 2003;188:1383-92.

39. Wacholder S. Chapter 18: statistical issues in the design and analysis of studies of human papillomavirus and cervical neoplasia. J Natl Cancer Inst Monogr 2003;31:125-30.

40. Wright Jr TC, Massad LS, Dunton CJ, Spitzer M, Wilkinson EJ, Solomon D, et al. 2006 consensus guidelines for the management of women with cervical intraepithelial neoplasia or adenocarcinoma in situ. Am J Obstet Gynecol 2007;197:340-5.

41. Schiffman M, Rodríguez AC. Heterogeneity in CIN3 diagnosis. Lancet 2008;9:404-5.

42. Castellsagué X, Díaz M, De Sanjosé S, Muñoz N, Herrero R, Franceschi S, et al. Worldwide human papillomavirus etiology of cervical adenocarcinoma and its cofactors: implications for screening and prevention. J Natl Cancer Inst 2006;98:303-15. 
43. Freemantle N, Calvert M, Wood J, Eastaugh J, Griffin C. Composite outcomes in randomized trials: greater precision but with greater uncertainty? JAMA 2003;289:2554-9.

44. Montori VM, Permanyear-Miralda G, FerreiraGonzález I, Busse JW, Pacheco HV, et al. Validity of composite end points in clinical trials. BMJ 2005;330:594-6.

45. Lazcano E, Salazar E, Gutiérrez P, Aneles A, Hernández A, Viramontes J. Ensayos clínicos aleatorizados: variantes, métodos de aleatorización, análisis, consideraciones éticas y regulación. Salud Pública Méx 2004;46:559-84

46. Montori VM, Guyatt GH. Intention-to-treat principle. CMAJ 2001;165:1339-41.

47. Gluud LL. Bias in Clinical Intervention Research. Am J Epidemiol 2006;163:493-501.

48. Manriquez J, Valdivia G; Rada G, Letelier LM. Análisis crítico de ensayos Clínicos randomizados publicados en revistas biomédicas chilenas. Rev med Chile 2005;133:439-46.

49. Hollis S, Campbell F. What is meant by intention to treat analysis? Survey of published randomised controlled trials. BMJ 1999;319:670-4.

50. Weinfur KP, Hall MA, King NM, Friedman JY, Schulman KA and Sugarman J. Disclosure of financial relationships to participants in clinical research. NEJM 2009;361:916-21.

51. Doval HC. ¿Es fiable lo que leemos en las revistas médicas? resultados diferentes según el patrocinio de los ensayos clínicos. Revista Argentina de Cardiología 2007; 75:498-502.
52. Jørgensen AW, Hilden J, Gøtzsche PC. Cochrane reviews compared with industry supported metaanalyses and other meta-analyses of the same drugs: systematic Review. BMJ 2006;333:782.

53. Dibarbora, Elisa A. Ética de la Investigación en salud: Un giro copernicano en la relación sanitaria. Visitado en 2009 Ago 27. Disponible en: http://www. unesco.org.uy/shs/fileadmin/ templates/shs/archivos/ TrabajosLibres-Bioetica/6.\%20Etica\%20de\%20la \%20Investigacion\%20en\%20Salud.pdf

54. Slade BA, Leidel L, Vellozzi C, Woo EJ, Hua W, Sutherland A, et al. Postlicensure safety surveillance for quadrivalent human papillomavirus recombinant vaccine. JAMA 2009;302:750-7.

55. Brisson M, Van de Velde N, De Wals P, Boily M. Estimating the number needed to vaccinate to prevent diseases and death related to human papillomavirus infection. CMAJ 2007;177:464-8.

56. Hutchinson DJ, Klein KC. Human papillomavirus disease and vaccines. Am J Health Syst Pharm 2008;65:2105-12.

57. Winkler JL, Wittet S, Bartolini RM, CreedKanashiro HM, Lazcano-Ponce E, Lewis-Bell K, et al. Determinants of human papillomavirus vaccine acceptability in Latin America and the Caribbean. Vaccine 2008;26S: L73-9.

58. Sarin R. HPV vaccine for primary prevention of cervical cancer in developing countries: the missing links. J Can Res Ther 2008;4:105-6. 